\title{
Correction to: Statistical Analysis of Solar Events Associated with Storm Sudden Commencements over One Year of Solar Maximum During Cycle 23: Propagation from the Sun to the Earth and Effects
}

\author{
K. Bocchialini ${ }^{1}$ (D) B. Grison ${ }^{2} \cdot$ M. Menvielle ${ }^{3,4} \cdot$ A. Chambodut ${ }^{5}$. \\ N. Cornilleau-Wehrlin ${ }^{6,7}$ - D. Fontaine ${ }^{7}$ - A. Marchaudon $^{8,9} \cdot$ M. Pick $^{6}$ - F. Pitout ${ }^{8,9}$. \\ B. Schmieder ${ }^{6}$ - S. Régnier ${ }^{10}$ - I. Zouganelis ${ }^{11}$ \\ Published online: 3 April 2019 \\ (C) Springer Nature B.V. 2019
}

\section{Correction to: Solar Phys (2018) 293:75 \\ https://doi.org/10.1007/s11207-018-1278-5}

\begin{abstract}
Taking the 32 storm sudden commencements (SSCs) listed by the International Service of Geomagnetic Indices (ISGI) of the Observatory de l'Ebre during 2002 (solar ac-
\end{abstract}

Earth-affecting Solar Transients

Guest Editors: Jie Zhang, Xochitl Blanco-Cano, Nariaki Nitta, and Nandita Srivastava

The online version of the original article can be found under https://doi.org/10.1007/s11207-018-1278-5.

K. Bocchialini

karine.bocchialini@ias.u-psud.fr

1 Institut d'Astrophysique Spatiale, Univ. Paris-Sud, CNRS, Université Paris-Saclay, Bâtiment 121, 91405 Orsay CEDEX, France

2 Institute of Atmospheric Physics CAS, Bocni II, 1401, 14131 Prague 4, Czech Republic

3 CNRS, Laboratoire Atmosphères, Milieux, Observations Spatiales, Université Versailles Saint Quentin, Guyancourt, France

4 Département des Sciences de la Terre, Univ. Paris Sud, 91405 Orsay CEDEX, France

5 Institut de Physique du Globe de Strasbourg, UMR7516, CNRS, Université de Strasbourg/EOST, 5 rue René Descartes, 67084 Strasbourg CEDEX, France

6 Observatoire de Paris, LESIA, PSL Research University, 5 place Jules Janssen, 92195 Meudon CEDEX, France

7 LPP, CNRS, Ecole Polytechnique, UPMC Univ. Paris 06, Univ. Paris Sud, Observatoire de Paris, Université Paris-Saclay, Sorbonne Universités, PSL Research University, Ecole Polytechnique, 91128 Palaiseau CEDEX, France

8 Institut de Recherche en Astrophysique et Planétologie, Université de Toulouse, Toulouse, France

9 CNRS, UMR 5277, 9 Av. du Colonel Roche, BP 44346, 31028 Toulouse CEDEX 4, France

10 Department of Mathematics, Physics and Electrical Engineering, Northumbria University, Newcastle upon Tyne, NE1 8ST, UK 
tivity maximum in Cycle 23) as a starting point, we performed a multi-criterion analysis based on observations (propagation time, velocity comparisons, sense of the magnetic field rotation, radio waves) to associate them with solar sources, identified their effects in the interplanetary medium, and looked at the response of the terrestrial ionized and neutral environment. We find that $28 \mathrm{SSCs}$ can be related to 44 coronal mass ejections (CMEs), 15 with a unique CME and 13 with a series of multiple CMEs, among which 19 (68\%) involved halo CMEs. Twelve of the 19 fastest CMEs with speeds greater than $1000 \mathrm{~km} \mathrm{~s}^{-1}$ are halo CMEs. For the $44 \mathrm{CMEs}$, including 21 halo CMEs, the corresponding X-ray flare classes are: 4 X-class, $19 \mathrm{M}$-class, and $21 \mathrm{C}$-class flares. The probability for an SSC to occur is $75 \%$ if the CME is a halo CME. Among the 500, or even more, front-side, non-halo CMEs recorded in 2002, only 23 could be the source of an SSC, i.e. 5\%. The complex interactions between two (or more) CMEs and the modification of their trajectories have been examined using joint white-light and multiple-wavelength radio observations. The detection of longlasting type IV bursts observed at metric-hectometric wavelengths is a very useful criterion for the CME-SSC association. The events associated with the most depressed Dst values are also associated with type IV radio bursts. The four SSCs associated with a single shock at L1 correspond to four radio events exhibiting characteristics different from type IV radio bursts. The solar-wind structures at L1 after the $32 \mathrm{SSCs}$ are 12 magnetic clouds (MCs), 6 interplanetary coronal mass ejections (ICMEs) without an MC structure, 4 miscellaneous structures, which cannot unambiguously be classified as ICMEs, 5 corotating or stream interaction regions (CIRs/SIRs), one CIR caused two SSCs, and 4 shock events; note that one CIR caused two SSCs. The 11 MCs listed in 3 or more MC catalogs covering the year 2002 are associated with SSCs. For the three most intense geomagnetic storms (based on Dst minima) related to MCs, we note two sudden increases of the Dst, at the arrival of the sheath and the arrival of the MC itself. In terms of geoeffectiveness, the relation between the CME speed and the magnetic-storm intensity, as characterized using the Dst magnetic index, is very complex, but generally CMEs with velocities at the Sun larger than $1000 \mathrm{~km} \mathrm{~s}^{-1}$ have larger probabilities to trigger moderate or intense storms. The most geoeffective events are MCs, since $92 \%$ of them trigger moderate or intense storms, followed by ICMEs (33\%). At best, CIRs/SIRs only cause weak storms. We show that these geoeffective events (ICMEs or MCs) trigger an increased and combined auroral kilometric radiation (AKR) and nonthermal continuum (NTC) wave activity in the magnetosphere, an enhanced convection in the ionosphere, and a stronger response in the thermosphere. However, this trend does not appear clearly in the coupling functions, which exhibit relatively weak correlations between the solar-wind energy input and the amplitude of various geomagnetic indices, whereas the role of the southward component of the solar-wind magnetic field is confirmed. Some saturation appears for Dst values $<-100 \mathrm{nT}$ on the integrated values of the polar and auroral indices.

11 European Space Agency, ESAC, Madrid, Spain 
Table 3 The 44 CMEs associated with the 32 SSCs mentioned in Table 2: 21 halo CMEs (CMEH), 9 partialhalo CMEs (CMEP), and 14 non-halos CMEs (CMEN), detected in 2002 with an identified source at the Sun. Starting from the second column on, we list the date and time of the beginning of the solar event at the origin of the CME as seen in SOHO/EIT images at $30.4 \mathrm{~nm}$ or $19.5 \mathrm{~nm}$, source coordinates in seconds of arc from Sun center $([0,0])$ with positive to the west and north, and nature of the source seen in SOHO/EIT (AR $=$ active region, $\mathrm{Fi}=$ filament, $\mathrm{FL}=$ flare, $\mathrm{CH}=$ coronal hole), the final height $(h)$ in units of solar radii in the SOHO/LASCO FOV at which the CME is observed, the corresponding velocity $\left(V_{\odot}\right)\left[\mathrm{km} \mathrm{s}^{-1}\right]$, the acceleration $(a)\left[\mathrm{m} \mathrm{s}^{-2}\right]$, the exit time from LASCO FOV, and the flare class (GOES).

\begin{tabular}{|c|c|c|c|c|c|c|c|c|c|}
\hline \multirow{2}{*}{$\begin{array}{l}\text { CME } \\
\text { No. }\end{array}$} & \multicolumn{4}{|c|}{$\underline{\text { Source (EIT) }}$} & \multicolumn{4}{|c|}{ CME (LASCO) } & \multirow{2}{*}{$\begin{array}{l}\text { Flare } \\
\text { class }\end{array}$} \\
\hline & Date & $\begin{array}{l}\text { Time } \\
{[\mathrm{UT}]}\end{array}$ & Coord. & Source type & $\bar{h}$ & $V_{\odot}$ & $a$ & $\begin{array}{l}\text { Exit time } \\
\text { [UT] }\end{array}$ & \\
\hline CMEH01 & 27 Jan & $12: 24$ & $(375,850)$ & $\mathrm{AR}$ & 26 & 1000 & -19.2 & $16: 30$ & $\mathrm{C}$ \\
\hline CMEN02 & $28 \mathrm{Jan}$ & $9: 35$ & $(-200,-500)$ & $\mathrm{AR}, \mathrm{Fi}$ & 12 & 738 & 35.0 & $13: 00$ & $\mathrm{C}$ \\
\hline CMEN03 & $24 \mathrm{Feb}$ & $14: 45$ & $(650,-400)$ & $\mathrm{AR}, \mathrm{Fi}$ & 6 & 258 & 5.2 & $17: 50$ & $\mathrm{C}$ \\
\hline CMEH04 & $15 \mathrm{Mar}$ & $21: 48$ & $(113,-48)$ & $\mathrm{AR}, \mathrm{Fi}, \mathrm{CH}$ & 30 & 784 & -17.4 & $4: 30(+1)$ & M \\
\hline CMEP05 & 17 Mar & $10: 28$ & $(-273,-233)$ & $\mathrm{AR}, \mathrm{Fi}$ & 30 & 931 & -6.0 & $15: 45$ & M \\
\hline CMEH06 & $18 \mathrm{Mar}$ & $1: 48$ & $(410,-240)$ & $\mathrm{AR}, \mathrm{Fi}$ & 20 & 971 & -2.9 & $6: 30$ & M \\
\hline CMEP07 & 19 Mar & $9: 24$ & $(770,-70)$ & $\mathrm{AR}, \mathrm{Fi}$ & 28 & 711 & -0.9 & $16: 10$ & M \\
\hline CMEP08 & 19 Mar & $11: 12$ & $(770,-70)$ & $\mathrm{AR}, \mathrm{Fi}$ & 12 & 1030 & 46.4 & $13: 45$ & M \\
\hline CMEP09 & $20 \mathrm{Mar}$ & $23: 24$ & $(870,-270)$ & $\mathrm{AR}, \mathrm{Fi}$ & 30 & 1075 & -0.2 & $4: 50(+1)$ & $\mathrm{C}$ \\
\hline CMEH10 & $22 \mathrm{Mar}$ & $10: 36$ & $(980,-160)$ & $\mathrm{AR}, \mathrm{Fi}$ & 18 & 1685 & -22.5 & $12: 40$ & $\mathrm{C}$ \\
\hline CMEH11 & $22 \mathrm{Mar}$ & $11: 36$ & $(980,-160)$ & $\mathrm{AR}, \mathrm{Fi}$ & 36 & 1027 & 14.6 & $18: 30$ & M \\
\hline CMEN12 & $11 \mathrm{Apr}$ & $16: 24$ & $(420,-200)$ & $\mathrm{AR}, \mathrm{Fi}$ & 20 & 497 & -3.4 & $23: 30$ & $\mathrm{C}$ \\
\hline CMEH13 & $15 \mathrm{Apr}$ & $3: 12$ & $(252,-159)$ & AR & 26 & 742 & 2.1 & $9: 45$ & M \\
\hline CMEN14 & $16 \mathrm{Apr}$ & 11:00 & $(910,-216)$ & $\mathrm{AR}, \mathrm{Fi}$ & 15 & 421 & -9.1 & $15: 45$ & $\mathrm{C}$ \\
\hline CMEH15 & 17 Apr & $7: 50$ & $(550,-130)$ & $\mathrm{AR}, \mathrm{Fi}$ & 28 & 1103 & -19.7 & $12: 20$ & M \\
\hline CMEH16 & $21 \mathrm{Apr}$ & $00: 48$ & $(916,-229)$ & $\mathrm{AR}$ & 25 & 2,388 & -1.4 & $3: 20$ & $\mathrm{X}$ \\
\hline CMEN17 & 06 May & $23: 47$ & $(800,400)$ & $\mathrm{AR}$ & 28 & 1266 & 5.6 & $4: 10(+1)$ & $\mathrm{C}$ \\
\hline CMEH18 & 07 May & $3: 36$ & $(-214,-109)$ & $\mathrm{AR}, \mathrm{Fi}$ & 6 & 926 & 158.1 & $4: 50$ & $\mathrm{C}$ \\
\hline CMEH19 & 08 May & $13: 13$ & $(130,-150)$ & $\mathrm{AR}, \mathrm{Fi}$ & 5 & 697 & 78.9 & $14: 30$ & $\mathrm{C}$ \\
\hline CMEH20 & 15 May & $23: 47$ & $(-197,-316)$ & AR & 28 & 506 & -6.6 & $8: 45(+1)$ & $\mathrm{C}$ \\
\hline CMEN21 & 17 May & $00: 47$ & $(-100,-350)$ & $\mathrm{Fi}$ & 20 & 532 & 5.5 & $8: 30$ & $\mathrm{C}$ \\
\hline CMEN22 & 17 May & $7: 48$ & $(-900,200)$ & AR & 15 & 616 & -7.5 & $11: 40$ & M \\
\hline CMEN23 & 18 May & $11: 50$ & $(-388,-455)$ & AR & 6 & 614 & 45.6 & $14: 00$ & $\mathrm{C}$ \\
\hline CMEP24 & 21 May & $23: 24$ & $(881,-319)$ & $\mathrm{AR}, \mathrm{Fi}$ & 28 & 1341 & 14.2 & $3: 45(+1)$ & $\mathrm{C}$ \\
\hline CMEH 25 & 22 May & $03: 12$ & $(881,-319)$ & $\mathrm{AR}, \mathrm{Fi}$ & 28 & 1504 & -10.4 & $6: 50$ & $\mathrm{C}$ \\
\hline CMEP26 & 27 May & $12: 23$ & $(-250,400)$ & $\mathrm{Fi}$ & 18 & 1122 & 3.8 & $15: 50$ & $\mathrm{C}$ \\
\hline $\mathrm{CMEH} 27$ & $15 \mathrm{Jul}$ & $19: 59$ & $(15,239)$ & $\mathrm{AR}, \mathrm{Fi}$ & 28 & 973 & -25.6 & $0: 20(+1)$ & $\mathrm{X}$ \\
\hline CMEP28 & $15 \mathrm{Jul}$ & 21:00 & $(15,239)$ & $\mathrm{AR}, \mathrm{Fi}$ & 23 & 1264 & -7.3 & $0: 20(+1)$ & M \\
\hline CMEH29 & $18 \mathrm{Jul}$ & $7: 59$ & $(500,250)$ & $\mathrm{AR}, \mathrm{FL}$ & 22 & 919 & -30.1 & $11: 20$ & $\mathrm{X}$ \\
\hline CMEP30 & $18 \mathrm{Jul}$ & $11: 30$ & $(-730,200)$ & AR & 23 & 680 & -14.0 & $16: 20$ & $\mathrm{C}$ \\
\hline СМЕH31 & $18 \mathrm{Jul}$ & $18: 26$ & $(-730,200)$ & $\mathrm{AR}$ & 28 & 1788 & - & $21: 20$ & $\mathrm{C}$ \\
\hline СMEH32 & $26 \mathrm{Jul}$ & $21: 12$ & $(-400,-370)$ & $\mathrm{AR}, \mathrm{Fi}$ & 30 & 816 & -0.1 & $4: 20(+1)$ & M \\
\hline CMEN33 & $29 \mathrm{Jul}$ & $02: 30$ & $(150,-350)$ & AR & 25 & 409 & 3.8 & $15: 00$ & M \\
\hline CMEN34 & $29 \mathrm{Jul}$ & $10: 59$ & $(150,-350)$ & AR & 11 & 301 & -3.8 & $16: 00$ & M \\
\hline CMEN35 & $30 \mathrm{Jul}$ & $00: 30$ & $(700,-700)$ & $\mathrm{Fi}$ & 15 & 998 & 32 & $4: 20$ & $\mathrm{C}$ \\
\hline СМЕН36 & 16 Aug & $11: 24$ & $(-250,-200)$ & $\mathrm{AR}, \mathrm{Fi}$ & 23 & 1239 & -67.1 & $15: 20$ & M \\
\hline
\end{tabular}


Table 3 (Continued)

\begin{tabular}{|c|c|c|c|c|c|c|c|c|c|}
\hline \multirow{2}{*}{$\begin{array}{l}\text { CME } \\
\text { No. }\end{array}$} & \multicolumn{4}{|c|}{ Source (EIT) } & \multicolumn{4}{|c|}{ CME (LASCO) } & \multirow{2}{*}{$\begin{array}{l}\text { Flare } \\
\text { class }\end{array}$} \\
\hline & Date & $\begin{array}{l}\text { Time } \\
{[U T]}\end{array}$ & Coord. & Source type & $\bar{h}$ & $V_{\odot}$ & $a$ & $\begin{array}{l}\text { Exit time } \\
{[\mathrm{UT}]}\end{array}$ & \\
\hline CMEP37 & 23 Aug & $05: 47$ & $(-243,-279)$ & $\mathrm{AR}$ & 8 & 622 & 36.6 & $8: 20$ & M \\
\hline СМЕH38 & $24 \mathrm{Aug}$ & $01: 13$ & $(950,-100)$ & AR & 26 & 2,066 & 43.7 & $3: 20$ & $\mathrm{X}$ \\
\hline СМЕН39 & 05 Sep & $16: 30$ & $(-400,23)$ & AR & 17 & 1855 & 43.0 & $18: 20$ & M \\
\hline CMEN40 & 27 Sep & $01: 36$ & $(900,-200)$ & $\mathrm{AR}$ & 21 & 1300 & -61 & $3: 40$ & M \\
\hline CMEN41 & 27 Sep & $13: 13$ & $(-650,150)$ & AR & 20 & 510 & -10 & $18: 45$ & M \\
\hline CMEN42 & $06 \mathrm{Nov}$ & $05: 24$ & $(-49,-280)$ & AR & 16 & 485 & -6.3 & 11:00 & $\mathrm{C}$ \\
\hline CMEH43 & 09 Nov & $12: 54$ & $(624,-205)$ & $\mathrm{AR}, \mathrm{Fi}$ & 26 & 1977 & 35.3 & $15: 40$ & M \\
\hline CMEH44 & $24 \mathrm{Nov}$ & $19: 13$ & $(-750,280)$ & $\mathrm{Fi}$ & 20 & 1179 & 20.5 & $23: 20$ & $\mathrm{C}$ \\
\hline
\end{tabular}

\subsection{Statistical Considerations on the Listed CMEs}

As mentioned in the previous section, we identify one or more possible solar sources for each SSC-led event. The probability for an SSC to occur is some $75 \%$ if the CME is a halo (21/28). According to the CDAW list, more than 500 non-halo, front-side CME were observed in 2002 ( 1.5 per day on average). Only 23 CMEs (see Table 3 ) could be the sources of an SSC, i.e. about $5 \%(23 / 500)$.

For the 44 CMEs associated with an SSC (as Leading or Contrib.) in Table 2,

- 18 have their source only in an AR (in one case, EIT detected the accompanying flare), 4 have their source only in a filament, and 22 have their source in an AR and a filament (including one case near a coronal hole $(\mathrm{CH})$ ). Thus $91 \%(40 / 44)$ of the CMEs have their source in an AR (with or without a filament), 60\% (26/44) in a filament (in or out of an AR).

- $73 \%(32 / 44)$ come from the southern hemisphere of the Sun, and $27 \%(12 / 44)$ from the northern hemisphere of the Sun.

- $39 \%(17 / 44)$ come from the eastern side of the Sun, 61\% (27/44) from the western side of the Sun, which is not surprising.

- $13.5 \%$ (6/44) have a velocity less than $500 \mathrm{~km} \mathrm{~s}^{-1}$ (non-halo CME), 43\% (19/44) a velocity ranging from $500 \mathrm{~km} \mathrm{~s}^{-1}$ to $999 \mathrm{~km} \mathrm{~s}^{-1}, 38.5 \%(17 / 44)$ a velocity between $1000 \mathrm{~km} \mathrm{~s}^{-1}$ and $2000 \mathrm{~km} \mathrm{~s}^{-1}$ (10/17 are halo CME), and 4\% (2/44) a velocity greater than $2000 \mathrm{~km} \mathrm{~s}^{-1}$ (2 halo CME),

- 21 CMEs are associated with GOES C-class events, 19 with M-class events, and 4 with X-class events. All but one are linked to an AR. In 2002, around 2000 C-class flares, around $200 \mathrm{M}$-class flares, and $12 \mathrm{X}$-class flares were seen by GOES.

Publisher's Note Springer Nature remains neutral with regard to jurisdictional claims in published maps and institutional affiliations. 


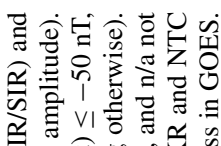

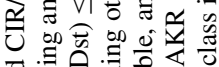

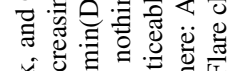

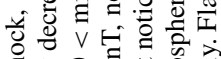

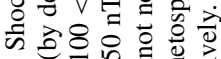

نํ.

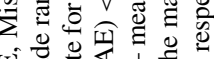

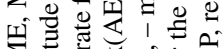

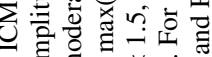

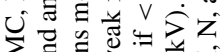

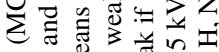

同导

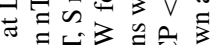

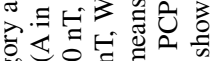

이요

苋艺

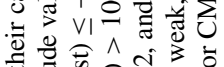

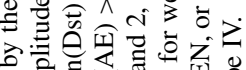

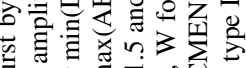

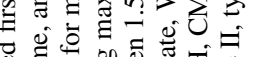

Ð ఏ जิ

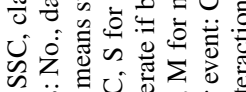

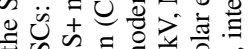
๙

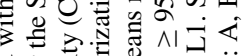

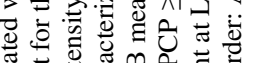

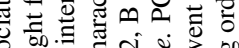
०. ปิ

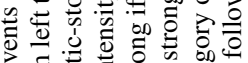
ठี. 巳

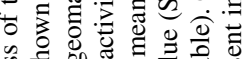

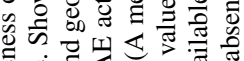
ญ

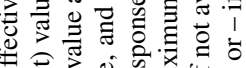

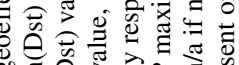

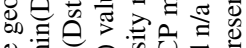

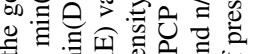

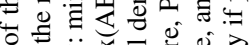

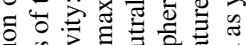

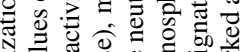

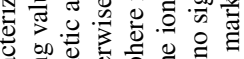

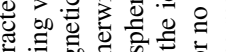

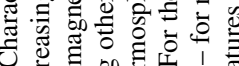
U.

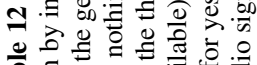

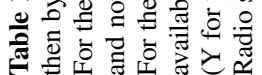

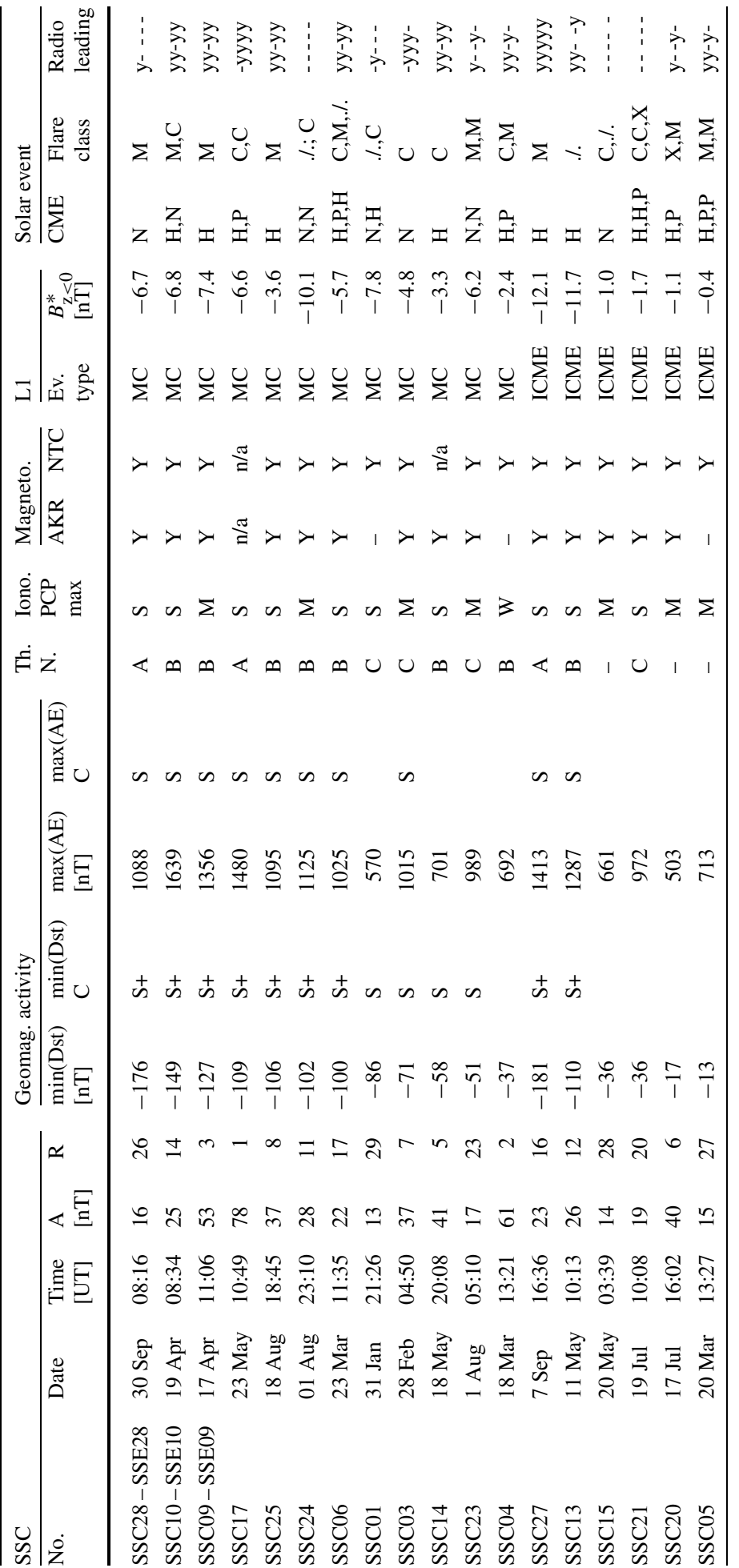




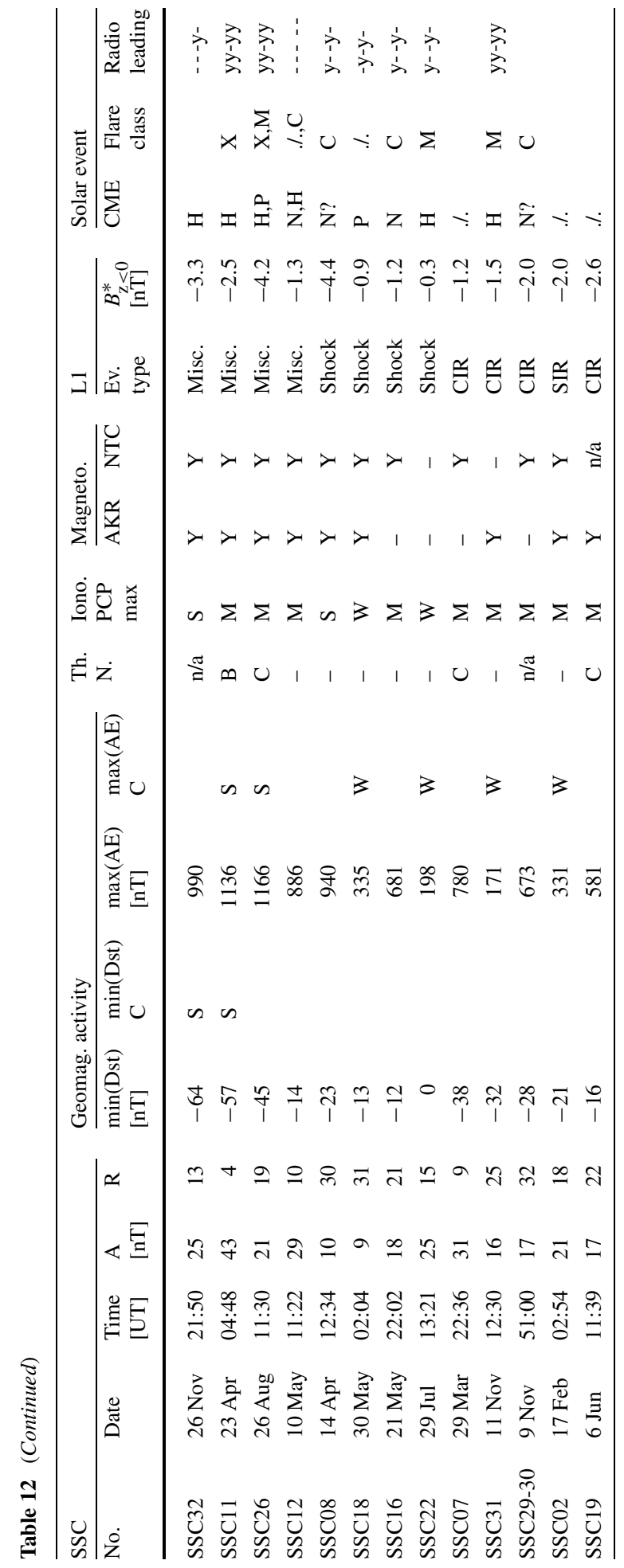

